\title{
FACILE GREEN FABRICATION AND OPTICAL STUDIES OF POLYANILINE /CUO DOPED NANOCOMPOSITES (NCS)
}

\author{
Hajeebaba K Inamdar ${ }^{1}$, Mahalesh Devendrappa ${ }^{2}$, Rajesh Siddanna ${ }^{3}$, M.V.N. Ambika Prasad ${ }^{4}$, \\ R D Mathad ${ }^{5}$ \\ ${ }_{1,2,3,4,5}$ Department of Post Graduate Studies and Research in Physics, Gulbarga University, Kalaburagi- 585 106, \\ Karnataka, India.
}

\begin{abstract}
This study reports the synthesis of Polyaniline/Cu-doped nanocomposites (NCs). Polyaniline/CuO -doped NCs were prepared by in situ polymerization of Aniline monomer in the presence of $\mathrm{CuO}$-doped NPs synthesized by solution combustion method using Aloe-vera gel as fuel with ammonium per sulfate (APS) as oxidant. Different concentrations of CuO (10-50 wt \%) NPs were ncorporated into the Polyaniline. The obtained final product was well characterized by means of PXRD, UV-Vis Spectroscophy, SEM, and transmission electron microscopy (TEM).The size of the NPs were calculated by Scherer and Williamson-Hall methods, and it was found to be around $20 \mathrm{~nm}$. TEM results are inconsistent with the XRD results. The morphology of the obtained product shows the porous and agglomerated particles which are due to large amount of gas evolution during NPS synthesis by solution combustion method. The present study demonstrates that the prepared samples are quite useful for electrochemical storage applications
\end{abstract}

Keywords: Bio-Mediated Synthesis, Polyaniline, $\mathrm{CuO}$, AC Conductivity, SEM.

\section{INTRODUCTION}

In recent years nanocomposites have become one of the most extensively studied materials all over the world, as they have shown to posseses several technological applications. Further, nanocomposites self-possessedaof conductingspolymers and metalsoxides have opened numerous applicationss e.g. in drugsrelease, conductive paints, rechargeablesbatteries, toners and smartswindows etc. [1-3]. The present study especially aims to investigate PANI-CuO nanocomposites in order to obtain a new noble material which can be utilized for Optical and electrical applications.

$\mathrm{CuO}$ is a semiconducting material that has a direct wide band gap of $2.16 \mathrm{eV}$ at room temperature. Indeed $\mathrm{CuO}$ is a peculiar material that exhibits multiple properties that include piezoelectric, semiconducting, pyroelectric and photo catalytic activities. In the present paper, PANI/CuO composites were prepared by in situ polymerization of aniline monomer with different doping concentrations of $\mathrm{CuO}$. All the composites have been analyzed using X-ray Diffractions(XRD) and Scanning Electron Microscopy (SEM). The dc conductivity of these composites was studied as a function asatemperature at different dopant concentrations.

\section{SYNTHESIS OF POLYANILINE}

The synthesis Polyaniline (PANI) was based on mixing liquid solution of aniline hydrochloride and APS at $32^{\circ} \mathrm{c}$ temperature, followed by taking part of Polanilin hydrochloride by filtration and drying. An equi-molar volume of aniline and hydrochloride acid was dissolved in distilled water in a beaker to obtain $100 \mathrm{ml}$ of solution. Similarly, ammonium per sulphate $(0.6 \mathrm{M})$ was dissolved in $100 \mathrm{ml}$ water. Both solutions were left 1 hour at room temperature and then mix in a beaker, stirred with a mechanical stirrer and allowed to polymerizing. After a day, the PANI precipitate was collected on a filter washed with $0.3 \mathrm{M} \mathrm{HCL}$ and acetone repeatedly. The Polyaniline hydrochloride powder was then kept at $60^{\circ} \mathrm{C}$ for 24 hours ${ }^{[2]}$.

\subsection{Bio -Mediated Preparation of $\mathrm{CuO}$ Nanoparticles}

The copper oxidesnanoparticles were synthesizedsby 'selfpropagatingslowstemperaturescombustionsmethod', employi ng Copper nitrate $\left.\left(\mathrm{Cu}\left(\mathrm{NO}_{3}\right)_{2} \cdot 6 \mathrm{H}_{2} \mathrm{O}\right)\right)$ as precursor and AloeVera gel as a fuel. In fact $2.95 \mathrm{~g}$ of copper nitrate was taken in $300 \mathrm{ml}$ petri-dish and $15 \mathrm{ml}$ of Aloe-Vera gel was added to the petri-dish and kept on a magnetic stirrer for $\sim 15 \mathrm{~min}$. The uniform mixture of both oxidizer as well as the fuel was then introduced into the pre-heated muffle furnace kept at $400{ }^{\circ} \mathrm{C}$. The mixture boils with froth yielding finally a black 
powder of $\mathrm{CuO}$ nanoparticles. The average particle size of the $\mathrm{CuO}$ was found by Debay Sherer Method to be $\sim 45$ $\mathrm{nm}^{[2]}$. The below figure shows the flow chart of sample preparation method

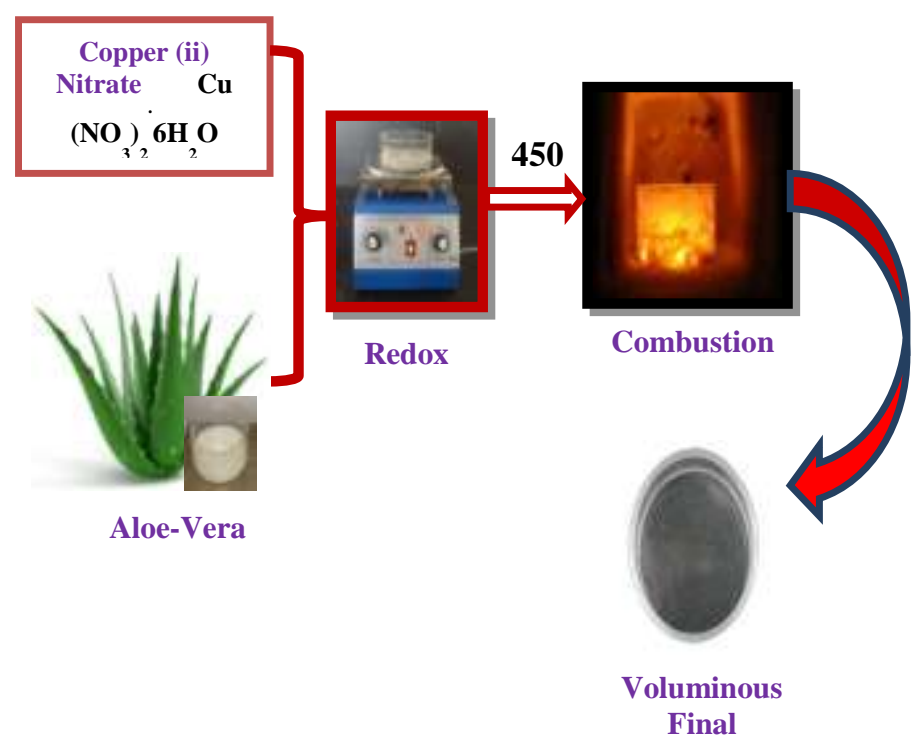

\subsection{Synthesis of Polyaniline/Copper Oxide Nano-}

\section{Composites}

Synthesis of Polyaniline-Copper oxidesnanocomposites werescarried out by Ssolution cast method. Anilines( $0.3 \mathrm{M})$ was mixed in $0.3 \mathrm{M} \mathrm{HClsand} \mathrm{stirred} \mathrm{for} 15$ minsto form aniline hydrochloride. Copper oxide nanoparticles were addedsin thesmass fraction to the above solution with vigorous mixing in orderato keepsthesCopper oxide homogeneouslyssuspendedsin thessolution. To thisssolution, $0.6 \mathrm{M}$ of APS, whichsactssas an oxidizerawas slowly added drop by drop withscontinuous mixing at icestemperature for 4 hours toscompletely polymerize. Thesprecipitateswas filtered, washed with deionized water and acetone, and finallysdriedain an oven fors $24 \mathrm{~h}$ to reached asconstant mass. The Polyaniline-Copper oxide nano-composites were thus obtained containing 50wt \%( i.e.50\%weight percentage of Copper oxide) $)^{[2]}$.

\section{XRD ANALYSIS}

X-Ray Diffraction were studies using Shimadzu-7000 diffractometer with $\mathrm{Cu}$ as the target $(1.54 \AA)$ and nickel as the filter. Fig.1 (a) Observed diffraction pattern of Polyaniline. A broad peak centered at $2 \theta=25.53^{\circ}$ may be assigned to the scattering from the Polyaniline chains at interplanar spacing which clearly implies the amorphous nature of Polyaniline and it corresponds to diffraction planes (200) of pure Polyaniline ${ }^{[2]}$.

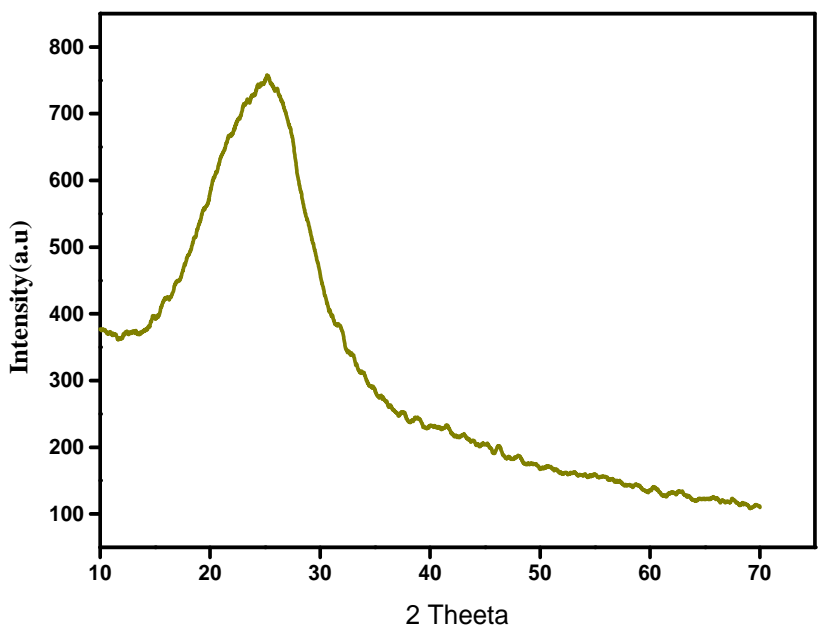

Fig -1: XRD Pattern of pure Polyaniline.

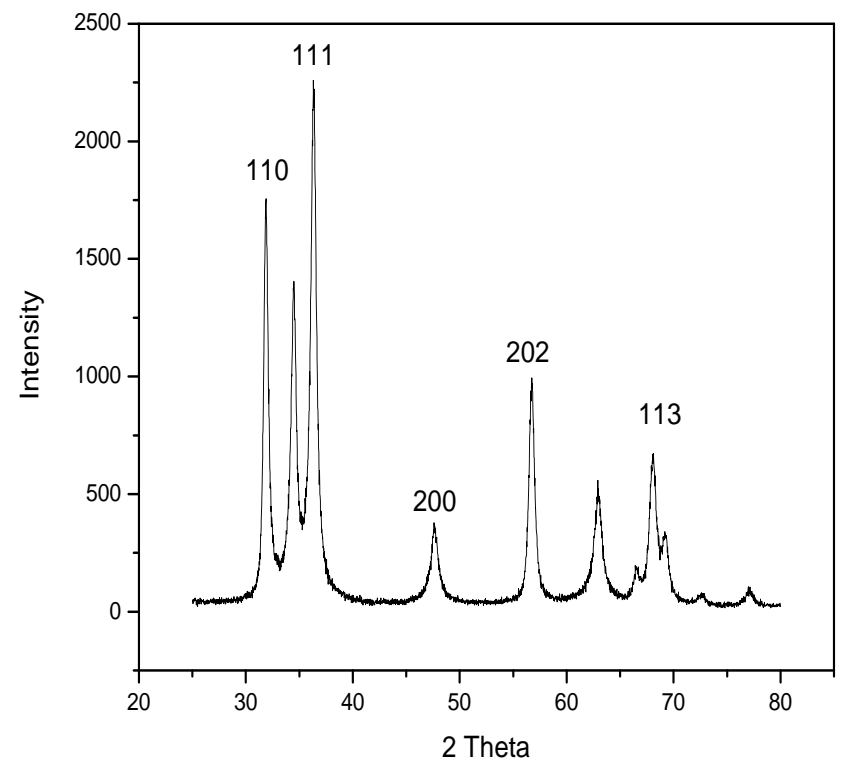

Fig -2: XRD Pattern of copper oxide nanoparticles.

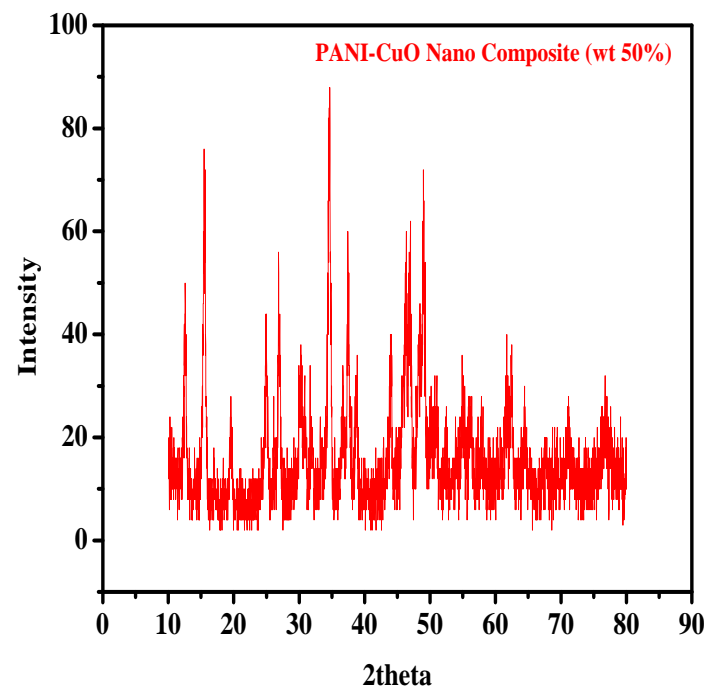

Fig -3: XRD Pattern of Polyaniline copper oxide nanocomposites. 
Fig. 2 Shows a broad peak at $2 \theta=16^{0}, 37^{0} \& 49.75^{\circ}$ which has a sharpsandswell definedspeaksandsit indicatessgood crystallinitysof $\mathrm{CuO}$ nanoparticles Fig. 3 showsstheaintensity of diffractionspeaksaforaPANI/CuO nanocomposites andsit issfoundstoabe lowerathan thatapure Copper oxide.The peaks of purescopper oxide nanoparticlesspresent in PANI/CuOsnanocomposites. ThesXRDspeakssaresbroad indicatingsthatstheeparticlesfare insnanometer size (JCPDS No.04-0835). The amorphoussbackgroundshumpscomes fromsthesPolyaniline. The average particle size of nickel oxide calculated by using Scherer equation is found to be $25 \mathrm{~nm}^{[5]}$.

\section{SEM MICROGRAPH}

Thesmorphologysofethernano copper oxidetand nanocompositesainathe form of powdersinvestigated using. SEM micrographs Fig 4.1 ( a) reveal that the pure $\mathrm{CuO}$ nano particles are irregular agglomerated in nature which is due to the evolution of large amount of gases during green combustion process. Fig 4.1 (b) shows SEM micrographs for PANI: $\mathrm{CuO}(50 \mathrm{wt} \%)$ nanocomposites ${ }^{[6,8]}$.

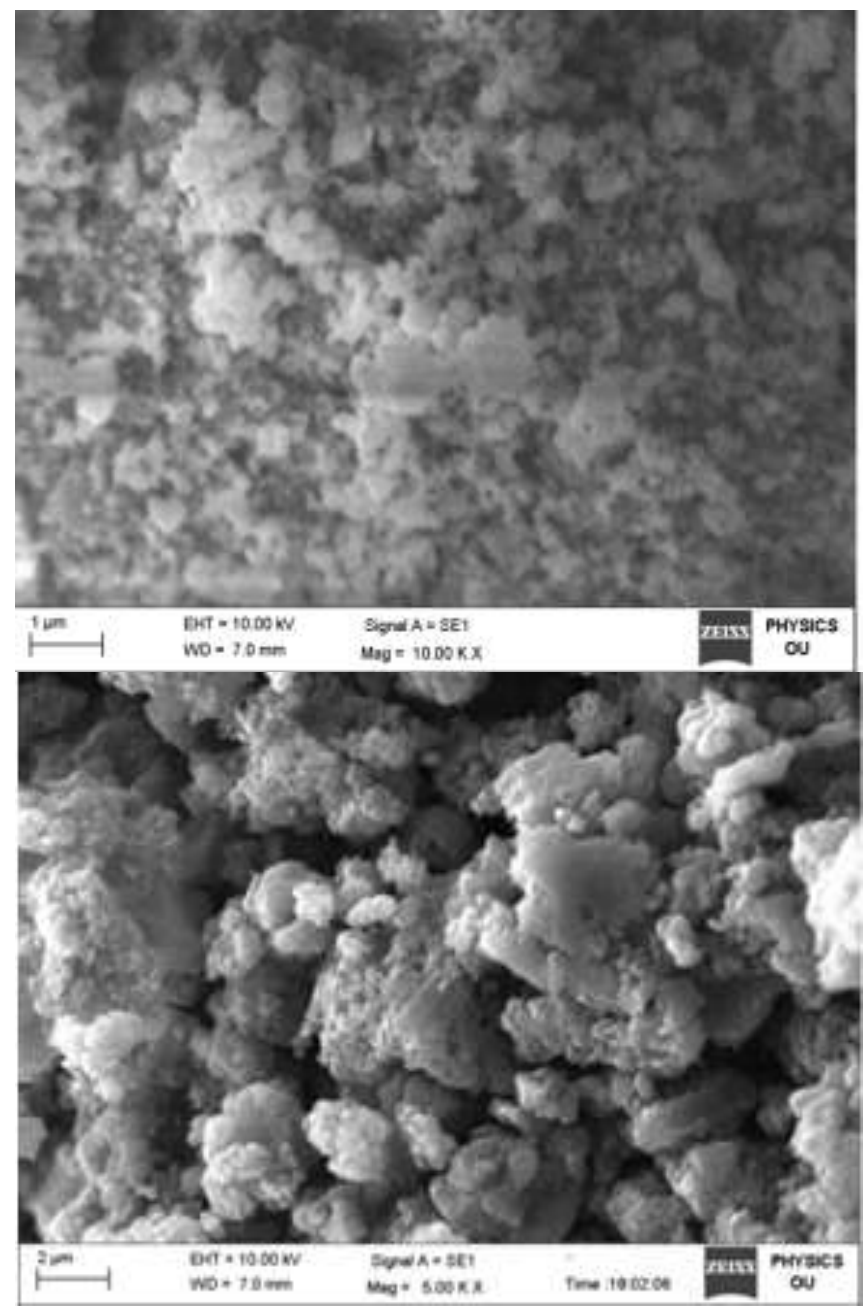

Fig -4: SEM Micrographs of Pure CuO Nanoparticles \&PANI: $\mathrm{CuO}$ (50 wt \%).

\section{UV-VISIBLE SPECTROSCOPIC STUDIES}

TheaUV-visible absorption spectra of Polyaniline , $\mathrm{CuO}$ NPs and Polyaniline- $\mathrm{CuO}$ nanocompositesswere recorded at room temperature are given in Fig.5 it reveals that distinctivespeaks of polyaniline appear at about $443 \mathrm{~nm}(2$. $\mathrm{eV}), 642 \mathrm{~nm}(1.93 \mathrm{eV})$ sand $916 \mathrm{~nm}(1.75 \mathrm{eV})$ which are attributed to the $\mathrm{p}-\mathrm{p}^{*}$, and p-polaron transitionsrespectively From above Fig. it can be seened that the characteristic peaks of Coper oxide nanoparticle at $391 \mathrm{~nm}(2.17 \mathrm{eV})$ and $485 \mathrm{~nm}(2.16 \mathrm{eV}) \mathrm{nm}$ which establishedswith reflectance of $\mathrm{CuO}$ and $\mathrm{UV}-$ visible spectra of Polyanilinei- $\mathrm{CuO}(50 \%)$.

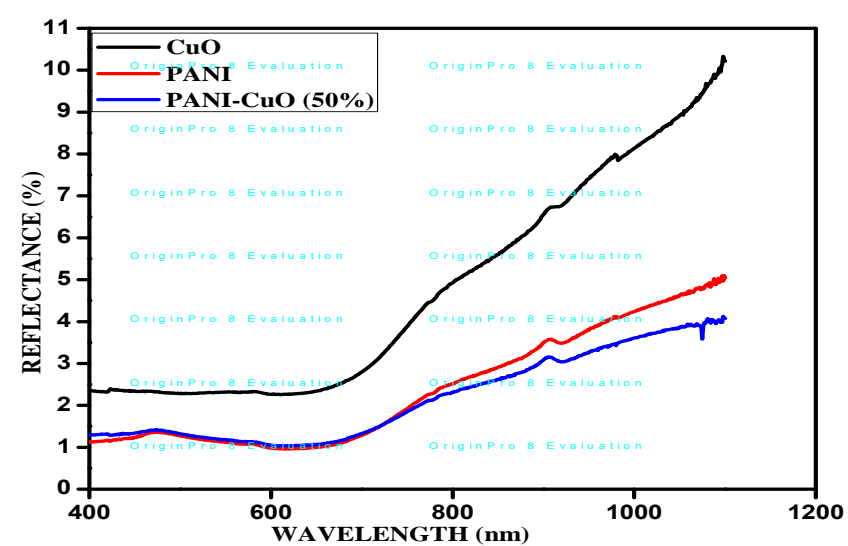

Fig -5: UV-visible spectroscopic graph of $\mathrm{CuO}$, PANI\& PANI-CuO.

\section{CONCLUSION}

The $\mathrm{CuO}$ Particles were synthesized by using Aloe Vera plant gel extract as a fuel by heat combustion method. The $\mathrm{CuO}$ nano particles were doped in Polyaniline (50 wt \%) synthesized using chemical polymerization process. The XRD shows the confirmation $\mathrm{CuO}$ Nanoparticles in PANI: $\mathrm{CuO}$ nanocomposites. The SEM micrographs reveal that the pure $\mathrm{CuO}$ nano particles are agglomerated in nature. UV Vis study reveals that the characteristicspeaks of $\mathrm{CuO}$ nanoparticlesat $391 \mathrm{~nm}(2.17 \mathrm{eV})$ and $485 \mathrm{~nm}(2.16 \mathrm{eV}) \mathrm{nm}$ whichsagreedswith reflectance of $\mathrm{CuO}$ and $\mathrm{UV}-$ visible spectra of PANI-CuO (50\%).

\section{ACKNOWLEDGEMENT}

The above said authors are greatly indebted UGC BSR Fellowship and also to Department of Physics, Gulbarga University, Kalaburagi.

\section{REFERENCES}

[1]. Morocco.J.Graphene-Based Polymer Nanocomposites Gerardo Martinez and Gary Ellis Institute of Polymer Science and Technology, Spanish National Research Council (CSIC) Spain.

[2]. Hajeebaba K Inamdar1,, H. Nagabhushana2, Basavaraja Sannakki1, R.D. Mathad1 * Materials Today: Proceedings 3 (2016) 3850-3854.

[3]. Rahman Khan, Y.K. Wee, W.A. Kamil Mahmood, Effects of $\mathrm{CuO}$ on the morphology and conducting properties of PANinanofibers. Synth Met 162, 1065 (2012). 
[4]. Satoshi Horikoshi and Nick Serpone Microwaves in Nanoparticle Synthesis, Introduction to Nanoparticles First Edition. Published 2013 by Wiley-VCH Verlag GmbH \& Co.KGaA.

[5]. Y.F. Lim, J.J. Choi, T. Hanrath, Facile synthesis of colloidal $\mathrm{CuO}$ nanocrystals for light-harvesting applications. J Nanomater 2012,6 (2012).

[6]. Liu C, Ma LP, Cheng HM (2010) Advanced materials for energy storage. Adv Mater 22(8):E28-E62.

[7].Sharanabasamma M Ambalagi*, Hajeebaba K Inamadar and Basavaraja Sannakki*, Materials Today: Proceedings 3 (2016) 3945-3950

[8]. David S.McLachlan and Godfrey Sauti: Journal of nanomaterials;2007 